\title{
Development of a new skin incision for conduct of conventional autopsy
}

\author{
Piyush Kapila ${ }^{\text {**}}$, Rahul Gupta', Sunil Kumar Raina², Aditya Kumar Sharma', Dhruv Gupta', Manav Sharma \\ and Vinod Kumar Bhardwaj
}

\begin{abstract}
Background: Since the practice of conventional autopsy, one new method for skin incision as "fourth incision" has been added to the preexisting conventional methods of skin incisions, i.e., "I"/"Y"/modified "Y". Reconstitution of conventional skin incisions leave visible defect (disfigurement) on the dead body which gets enhanced with invariable use of additional confirmatory or releasing incisions. Mutilation in form of disfigurement of the dead body is a major reason for legal heirs to withdraw the consent for pathological or clinical autopsy and is leading to worldwide significant drop in the rate of pathological autopsies. This study was formulated to develop a new course of skin incision for autopsy with the aim to minimize the visible defect and to obviate the need of extra incisions and so that it is time friendly for autopsy surgeons.

Result: On practicing this course of skin incision, the authors of study have noticed its advantages as it is cosmetically effective and time friendly and offers 100\% operational area with single incision in continuity in comparison to other advocated methods.

Conclusion: Its acceptability with its inherent advantages to autopsy surgeons and legal heirs of the dead body may increase the tendency to give desired informed consent for pathological autopsy. However, the validation of our method of skin incision in terms of different surgical and observer components is required to remove any bias regarding noticed advantages.
\end{abstract}

Keywords: Autopsy, Methods, Informed consent

\section{Background}

The conventional autopsy (a scientific tool to investigate the cause of death) remains "the gold standard" for advancement of medical knowledge (Burton and Underwood 2007). But its method is associated with many misconceptions and myths (Byard and Khong 1997). This has led to a drastic fall in the rate of pathological or clinical autopsies throughout the world. The most common reasons for refusal to give informed consent for autopsy include the fear of mutilation of the dead body, religious beliefs, and concerns about delays in funeral and removal of organs or body parts (Oluwasola et al. 2009).

The skin incision is an integral part of autopsy process to open the body. The reconstitution of the dead body produces visible defect (disfigurement) that becomes

\footnotetext{
*Correspondence: piyush.kapila@gmail.com

${ }^{1}$ Department of Forensic Medicine, IGMC Shimla (Himachal Pradesh), Shimla, India

Full list of author information is available at the end of the article
}

more pronounced with the use of additional confirmatory or releasing incisions.

An extensive search on PubMed reveals paucity of data on methods of skin incision for autopsy. Over the years, a few types (mainly three) of incisions are being recognized in forensic science.

In recent years, one new suggestion, i.e., "fourth incision," has been added to this list of existing conventional methods of skin incision ("I"/"Y"/modified "Y") (Table 1). Cosmetically effective fourth incision can help to alleviate fear for mutilation or disfigurement of legal heirs but is time consuming for autopsy surgeon (Patowary 2010).

The current study is aimed at the development of a new skin incision for autopsy (hereafter referred as "universal skin incision") for conduct of conventional autopsy. The idea is to minimize apparent disfigurement of dead body, reduce time consumption, and obviate the need of additional confirmatory or releasing incisions. 
Table 1 Characteristic features of the different types of incision

\begin{tabular}{|c|c|c|c|c|}
\hline Variable & I, $Y$, modified $Y$ & Fourth incision & Fifth incision & Remarks \\
\hline Length of incision & Less than fourth & More than conventional & Less than fourth & Advantage over fourth \\
\hline $\begin{array}{l}\text { Need for additional releasing or } \\
\text { confirmatory incisions }\end{array}$ & Yes & No & No & $\begin{array}{l}\text { Advantage over Conventional } \\
\text { while similar to fourth }\end{array}$ \\
\hline $\begin{array}{l}\text { Availability of operational area } \\
\text { for assessment without } \\
\text { additional incision }\end{array}$ & Less than $100 \%$ & $100 \%$ & $100 \%$ & $\begin{array}{l}\text { Advantage over Conventional } \\
\text { while similar to fourth }\end{array}$ \\
\hline $\begin{array}{l}\text { Visibility of reconstituted skin } \\
\text { incision on the front aspect } \\
\text { of the dead body }\end{array}$ & Yes & No & No & $\begin{array}{l}\text { Advantage over conventional } \\
\text { while similar to fourth }\end{array}$ \\
\hline Spillage of body fluid & Yes & No & No & $\begin{array}{l}\text { Advantage over Conventional } \\
\text { while similar to fourth }\end{array}$ \\
\hline Time taken to suture & Less & More than conventional & Less than fourth incision & Advantage over fourth \\
\hline Suturing the abdominal muscle flap & Not done & Done & Not required & Advantage over fourth \\
\hline
\end{tabular}

\section{Material and method \\ Development of incision}

The study was conducted at the Department of Forensic Medicine of a tertiary care teaching hospital in north India. For formulating the scale at initial stage, known anatomical landmarks for drawing up of the incision (hereafter incision points) were identified. The prepared list of these incision points was submitted to forensic experts to comment on their relevance for the proposed incision. The list was prepared after careful analysis of anatomical landmarks of already existing incision types. There was a general agreement among the experts for 15 incision points and two approaches (anterior and posterior). The incision points were named as templates. With every template, some alternatives were added and then resubmitted to a second set of forensic experts. It was pointed out that with every template, only two alternatives should be contained, and every alternative should be given a choice score or ranking in terms of preference (as first/second). The alternatives were developed to arrive at first choice templates. Any template or their alternatives, which were not approved by the experts (second set) or found to be insignificant in conduct of the incision, were subsequently discarded. The revised incision was submitted to the first set for identifying coherence in the revision. Thereafter, the universal incision was developed using anatomical landmarks which include the right and left mastoid area, inferior border of lower jaw, right acromion, mid-axillary line, right antero-superior iliac spine, right and left inguinal ligaments, left antero-superior iliac spine, and upper convex borders of gluteal region (Figs. 1 and 2). Once the landmarks and the approaches (anterior and posterior) were identified, the steps of incision were developed by the group of forensic experts (first and second) after due consultation.

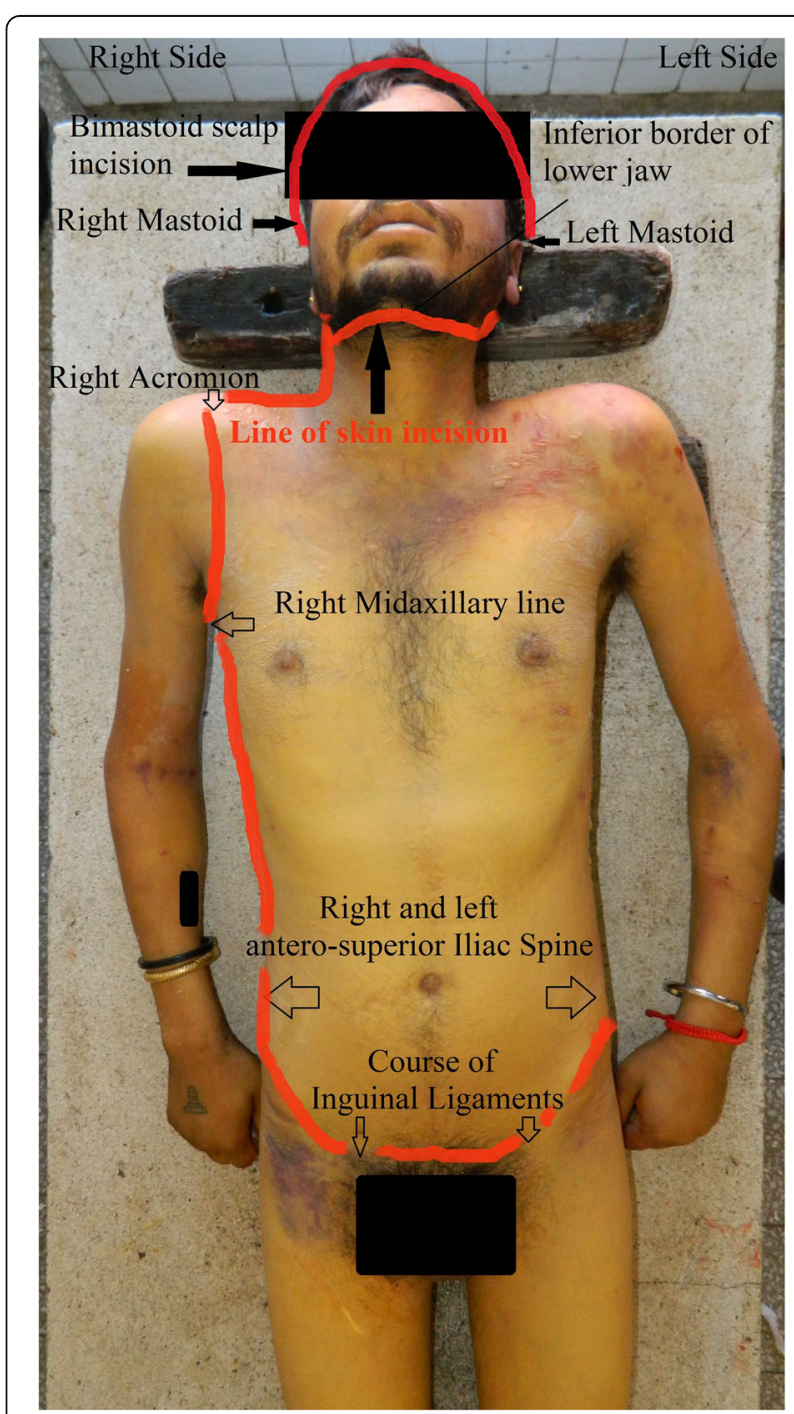

Fig. 1 Universal skin incision course with anatomical landmarks for anterior dissection during the autopsy 


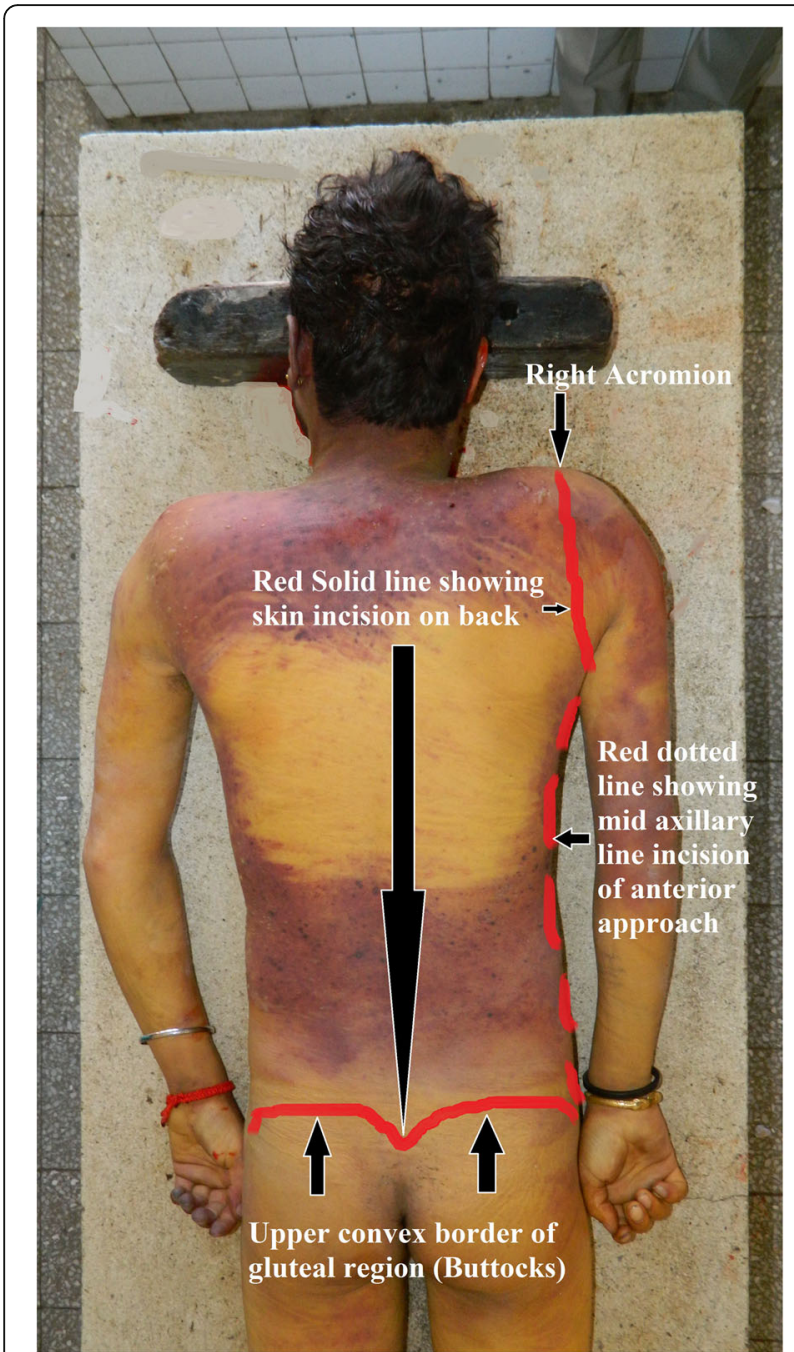

Fig. 2 Universal skin incision over the right shoulder's back and buttocks for posterior dissection during the autopsy

\section{Technical description of method for skin incision during autopsy}

A. Requirement: The incision requires the use of normal dissection instruments, routinely used in settings for autopsy: scalpel, toothed forceps, and ordinary curved scissor along with skin marker or gentian violet ink.

B. Steps of universal method of autopsy skin incision:

- For anterior approach to the dissection of the neck, chest, and abdomen:

1. The dead body has to be kept in a supine position with a wooden block under the posterior aspect of the shoulders to extend the neck.

2. A bi-mastoid incision in coronal plane one finger away from the attachment of the pinna has to be given on the scalp for opening the cranial cavity.

3. From the right end of the coronal bi-mastoid scalp incision line on the right mastoid area, a skin incision has to be given on the neck two fingers below to palpable inferior margin of the lower jaw to meet with its left end on the left mastoid area.

4. For opening the chest and abdominal cavity, the right end of bi-mastoid incision has to be extended downwards from the right mastoid area to pass over the right lateral side of the neck as posterior as possible to join at the right acromion (top of shoulder). From the right acromion, a curved skin incision has to be extended over the anterior surface near the medial border of the right shoulder joint till the mid-axillary line at level of the fourth rib, from where a vertical skin incision along the mid-axillary line has to be made downwards till the right antero-superior iliac spine.

5. From the right antero-superior iliac spine, the skin incision has to be extended bilaterally along the course of inguinal ligaments till the left antero-superior iliac spine.

6. Now, the scalpel blade has to be kept tangentially to the body surface to dissect free the anterior skin flap of the neck along with the anterior walls of the chest and abdomen from the underlying muscles and structures up to the left mid-axillary line without disrupting the anterior wall of the chest and abdominal cavity.

7. The anterior skin flap has to be pinned up on the left lateral side of the chest and abdomen by using clamps so that there is no restriction on complete view of the body during autopsy (Figs. 3 and 4).

8. The muscular flap of the anterior abdominal wall has to be excised with a scissor, starting from the anterior superior iliac spine along the course of both inguinal ligaments, till the left antero-superior iliac, then upwards along the left mid-axillary line till the tenth rib and the excision is turned towards the right-mid axillary line along both costal margins. This muscular flap has to be reflected towards the right abdominal flank (Fig. 5).

9. If the dissection on the back of the torso is not required, then after completing the examination of the neck and body cavities, the abdominal muscular flap is replaced to its 


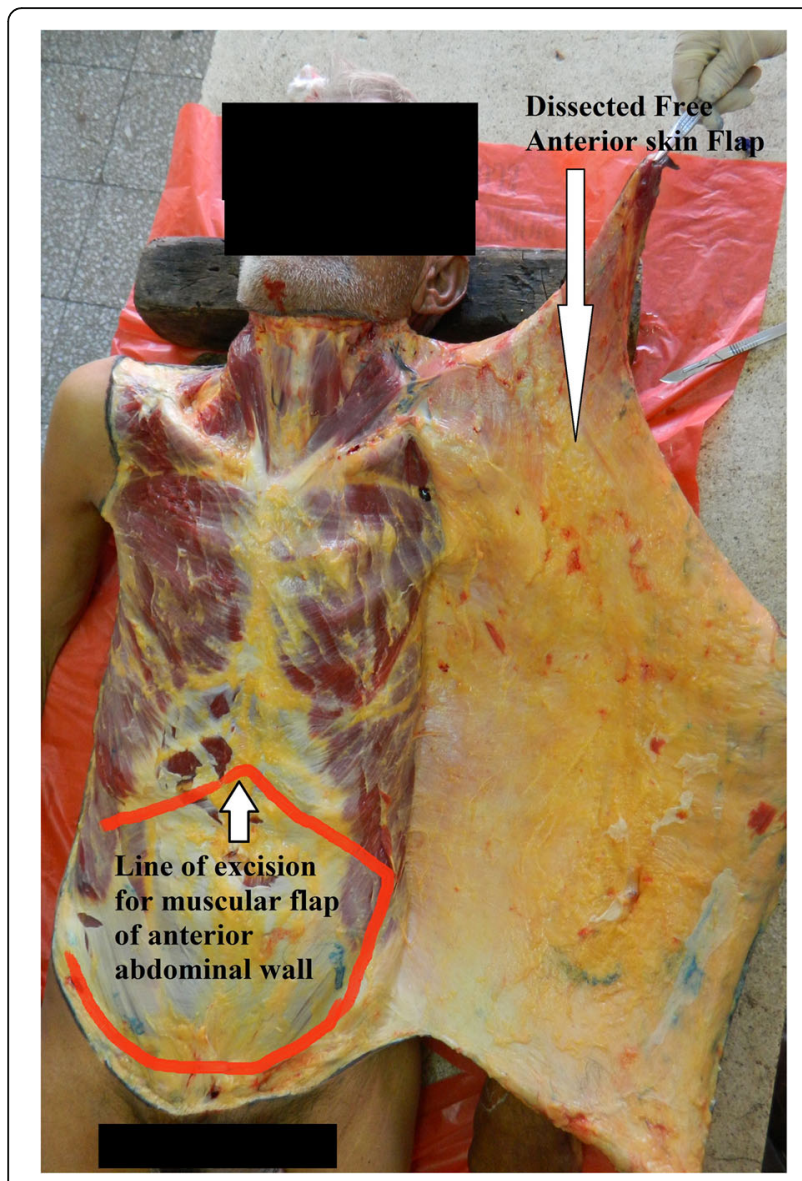

Fig. 3 Excision line for the abdominal muscular flap after reflecting the anterior skin flap towards the left lateral side

anatomical position. For reconstitution of the dead body, suturing of the skin incision is started from the left antero-superior spine to go over the incision on inguinal ligament then turning to the incision along the right mid-axillary line up to the right mastoid area followed by the over bi-mastoid incision, and in the end, suturing is carried from the left lower end of the bi-mastoid incision to its right lower end by stitching the jaw incision (Fig. 6).

- Steps for posterior approach to the dissection of the neck, chest, and abdomen:

The dissection of posterior aspect is sometimes needed in cases of death in custodial torture, trauma on the back, or abnormality of the spine and spinal cord.

1. The body has to be kept in a prone position by placing a wooden block below the anterior aspect of the shoulders for dorsal extension of the neck.

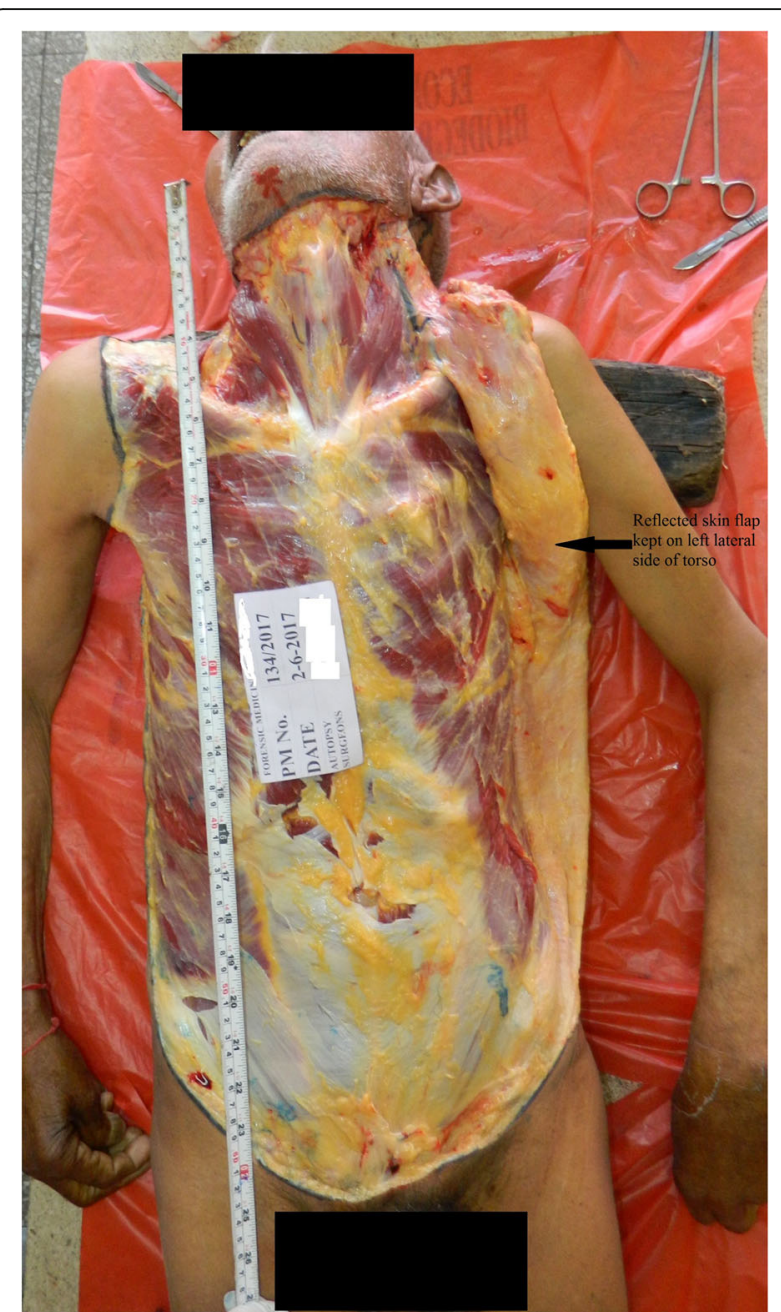

Fig. 4 The dissected free anterior skin flap rolled and clamped on the left lateral side of the torso

2. From the right acromion, a curved skin incision has to be extended over the posterior surface near the medial border of the right shoulder joint till the upper end of the vertical skin incision along the right mid-axillary line previously given for anterior approach.

3. One skin incision has to be given along the upper convex borders of the gluteal region (buttocks) from the right antero-superior iliac spine to the left antero-superior spine.

4. Now, with the scalpel blade while keeping it tangential to the body surface, the posterior skin flap of the neck and posterior walls of the chest and abdomen have to be dissected free from the underlying muscles and structures up to the left mid-axillary line.

5. The posterior skin flap has to be pinned up on the left lateral side of the chest and abdomen by using clamps so that there is no restriction on the complete view of the body during autopsy. 


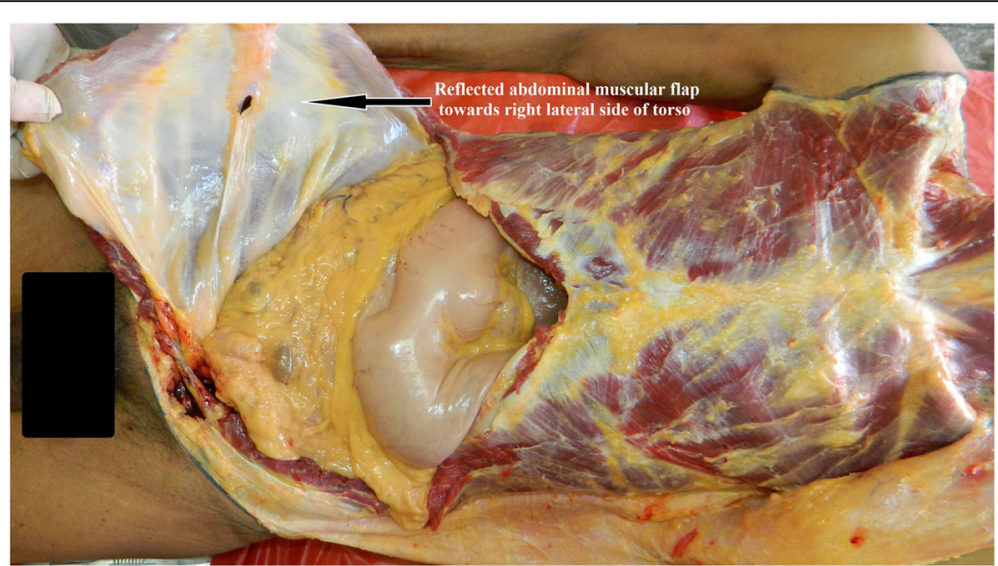

Fig. 5 After excising, the dissected free abdominal muscular flap reflected towards the right lateral side of the torso

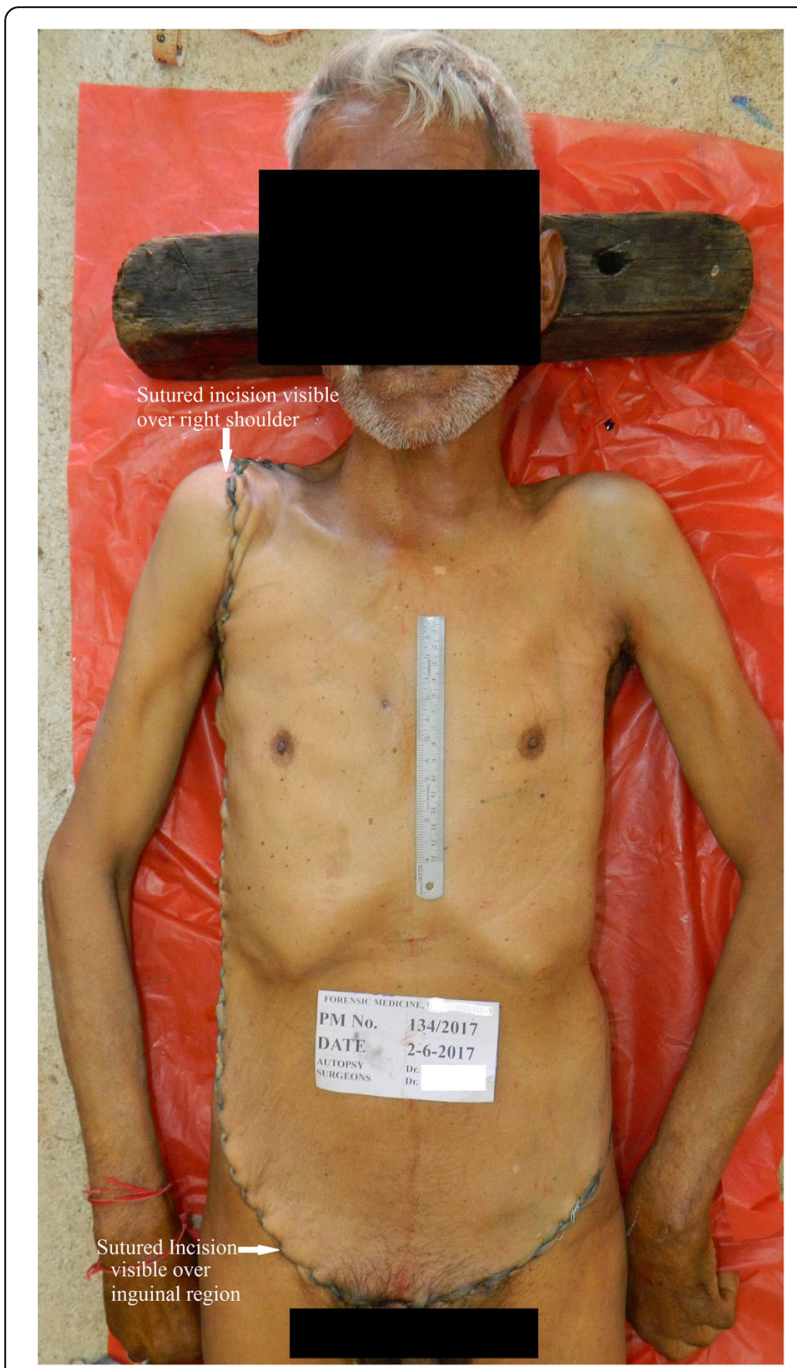

Fig. 6 On reconstitution, sutured incision visible on the right shoulder and inguinal region from frontal view
6. After examination of relevant posterior structures, the posterior skin flap has to be repositioned. Curved incisions over the back of the right shoulder and along convexity of the gluteal region are sutured separately.

\section{Precautions}

1. The line of the skin incision has to be marked first with a skin marker or gentian violet ink so that zig zag course of incision can be avoided.

2. The skin incision has to be made with a sharp new blade held perpendicular to the skin so that a chamfered skin incision resulting in an unsatisfactory reconstruction of the dead body can be avoided.

3. The scalpel blade has to be kept tangential to the body surface for dissecting free the anterior or posterior skin flap from the neck and torso.

4. The suturing for reconstruction of the dead body has to be done in the end if posterior dissection is required in a case.

\section{Results}

The results were evaluated using empirical evidence in terms of length of skin incision, need for additional releasing or confirmatory incisions, operational area for assessment, visibility of reconstituted skin incision on the front aspect of the dead body, spillage of body fluids, and time for suturing skin incision with regard to the conventional incisions, i.e., I, Y, modified $\mathrm{Y}$, and fourth incision. It was observed that the length of incision of our suggested incision on the front aspect of the dead body for anterior approach is less (equal to the length of the torso) than that of the fourth incision. The conventional incisions (I, Y, and modified Y) are shorter in length by width of the torso across inguinal ligaments. 
There is no need of additional incisions over the front and back of the neck along with the torso while adopting our suggested incision or fourth incision as compared to the autopsy conducted through conventional incisions. With the use of our suggested incision and fourth incision, the operational area for assessment of the neck and torso is about $100 \%$ without giving extra incisions, as is invariably required while performing autopsy through conventional incisions. After suturing, our suggested incision and fourth incision are not visible from direct front view of the neck and torso except at the right shoulder and inguinal region whereas sutured conventional incisions are visible on the front surface of the dead body. After reconstituting the dead body, spillage of fluids from the chest and abdominal cavities is almost nil while conducting autopsy with our suggested incision and fourth incision which is a common problem with conventional incisions. Time taken to suture is less in our suggested incision as compared to fourth incision, because of its shorter course, and the need of suturing the abdominal muscle flap is not required.

\section{Discussion}

The widely practiced conventional methods for skin incision to be made during autopsy process have been discussed in books devoted to forensic pathology. But while adopting one of the conventional methods of skin incision, separate incisions are invariably required for the assessment of soft tissue injury on the back and to open the spinal canal during autopsy. Further additional confirmatory (for differentiating bruise from hypostasis) or releasing incisions are required for complete exposure of the front of the neck (i.e., along the inferior border of the lower jaw and over the collar bones) and pelvic cavity (i.e., along the course of inguinal ligaments). On reconstitution of the dead body, sutured conventional incision on the front aspect of the body along with additional incisions leads to the visible artifacts over the skin surface, i.e., apparent disfigurement (mutilation) of the dead body. Therefore, these incisions are cosmetically ineffective and scare the legal heirs of the dead body.

Our proposed method for skin incision provides complete exposure of the whole circumference of the neck, thorax, and abdomen with single incision line; thereby, the operational area for autopsy assessment is almost $100 \%$. This aspect of our incision method is in corroboration with the use of fourth incision (Patowary 2010).

On reconstitution of the dead body after autopsy with any of conventional skin incisions, there is seepage of extravasated body fluids which leads to inconvenience during transportation to the attendants of the deceased. Adoption of the fourth incision method decreases the chance of seepage to almost nil as the abdominal cavity is closed first by stitching the rectus and then the flap of skin is replaced back covering it completely (Patowary 2010).

In our method of skin incision, seepage is nil too. However, there is no need to stitch the muscular flap of the anterior abdominal wall due to double breast advantage as the free end of the muscular flap is reflected back opposite to the line of skin closure.

In conventional methods, stitches made on the body for closing are seen on the front aspect of the body whereas in consonance with the fourth incision in our method too, the stitches except in the front of the shoulder and inguinal region are not apparent from the front or back view of the dead body.

The fourth incision consumes time, 10 min more for opening the body and 15 min more for closing, than in "I-shaped incision" (Patowary 2010).

In contrast, our method of skin incision is shorter in length that is equal to the length of the torso of the deceased as compared to fourth incision, and further, there is no need to stitch the muscular flap separately. Thereby, the extra time taken by fourth incision is almost curtailed by our method.

The current work presents a simple procedure of skin incision for the assessment of the neck, the front as well as back of the chest, and the abdomen and discusses its advantages on the basis of existing methodologies. The technique preserves the whole flap on the front and back of the neck, chest, and abdomen, and it does not interfere with the rest of the autopsy procedure. After dissection, reassessment of the wound tract in stab and firearm injury is easier and is convenient for surgical death cases too, as the proposed skin incision is not interfering with iatrogenic surgical incisions.

Our method is friendly for autopsy surgeons and legal heirs, as it is cosmetically effective and advocates single incision. Its routine application is both feasible and useful for autopsy on female corpse, cases of death due to custodial torture, trauma on back, surgical death cases, or fatal pressure over the neck. However, any new scientific method needs its unbiased validation of its procedure and outcome in light of the different aspects of doers and beneficiaries.

\section{Limitations}

The current study is based on a single case which may serve as a limitation to extrapolate findings to different settings. A validity study is being planned in the near future to establish the advantages of the new incision.

\section{Conclusions}

The sutured conventional skin incision on the front of the body along with sutured additional incisions leads to apparent disfigurement of the corpse. Though a new cosmetically effective fourth incision is advocated, it is 
time consuming. Our method of skin incision is time friendly as well as cosmetically effective and provides $100 \%$ operational area for autopsy surgeon to assess the head, neck, chest, and abdomen with single incision. To prove our new method of skin incision as a universal one, its validation in terms of different surgical (autopsy surgeons) and observer's (legal heirs of the dead body) components is required to remove any bias regarding its noticed advantages.

\section{Availability of data and materials}

Yes.

\section{Authors' contributions}

$P K, R G, S K R, A K S, D G, M S$, and VKB contributed to the concepts, definition of intellectual content, experimental studies, data acquisition, data analysis, manuscript editing and manuscript review and are the guarantors. PK, RG, and SKR contributed to the design, manuscript preparation, and literature search. All authors read and approved the final manuscript.

\section{Ethics approval and consent to participate}

Not applicable. As autopsy in cases of unnatural deaths is mandatory in this country and incisions are to be made, the care has been taken to conceal the identity of the dead body.

\section{Consent for publication}

Consent was taken from the legal heirs with condition of not revealing the identity of the dead body.

\section{Competing interests}

The authors declare that they have no competing interests.

\section{Publisher's Note}

Springer Nature remains neutral with regard to jurisdictional claims in published maps and institutional affiliations.

\section{Author details}

'Department of Forensic Medicine, IGMC Shimla (Himachal Pradesh), Shimla, India. ${ }^{2}$ Department of Community Medicine, DR. RPGMC, Tanda, Kangra, Himachal Pradesh, India.

Received: 25 December 2017 Accepted: 21 August 2018

Published online: 08 September 2018

\section{References}

Burton JL, Underwood J (2007) Clinical, educational, and epidemiological value of autopsy. Lancet 369:1471-1480

Byard RW, Khong TY (1997) Myths, misconceptions and the autopsy. Aust Fam Physician 26(5):555-557

Oluwasola OA et al (2009) The autopsy: knowledge, attitude, and perceptions of doctors and relatives of the deceased. Arch Pathol Lab Med 133:78-82

Patowary A (2010) The fourth incision: a cosmetic autopsy incision technique. Am J Forensic Med Pathol 31:37-41

\section{Submit your manuscript to a SpringerOpen ${ }^{\mathcal{O}}$ journal and benefit from:}

- Convenient online submission

- Rigorous peer review

- Open access: articles freely available online

- High visibility within the field

- Retaining the copyright to your article 